\title{
Synthesis of Sterically Protected Isoindoles from ortho-Phthalaldehyde
}

\author{
Michiyasu Nakao ${ }^{a}$ \\ Nanako Nishikioria \\ Akihito Nakamura ${ }^{a}$ \\ Murasaki Miyagia \\ Nao Shibata ${ }^{a}$ \\ Syuji Kitaike ${ }^{a}$ \\ Makoto Fukuib \\ Hiro-O Itob \\ Shigeki Sano*a \\ a Graduate School of Pharmaceutical Sciences, Tokushima \\ University, Sho-machi, Tokushima 770-8505, Japan \\ ssano@tokushima-u.ac.jp \\ ${ }^{\mathrm{b}}$ Department of Preventive Dentistry, Institute of Biomedical \\ Sciences, Tokushima University Graduate School, 3-18-15, \\ Kuramoto-cho, Tokushima 770-8504, Japan
}

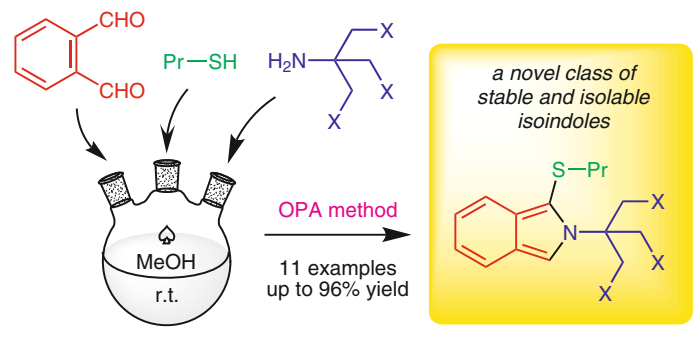

zation. ${ }^{6,7}$ However, from a synthetic point of view, the stabilization effects of these reagents are insufficient to isolate the resulting 1-alkylthio-2-alkyl-substituted isoindoles in pure forms. To the best of our knowledge, few reports are available on the synthesis of stable and isolable 1-alkylthio2-alkyl-substituted isoindoles based on the OPA method. In 2012, Sipos et al. reported the synthesis and biological activities of isoindole and benzoisoindole derivatives of glycopeptide antibiotics, which have large molecular sizes and function as primary amines in the OPA method. ${ }^{8}$ In this paper, we report on the synthesis of sterically protected 1-alkylthio-2-alkyl-substituted isoindoles using $C_{3}$-symmetric bulky amines derived from tris(hydroxyalkyl)aminomethane based on the OPA method. HPLC analysis of a mixture of stable isoindoles derived from alkyl thiols, OPA, and $O$-benzylated tris(hydroxypropyl)aminomethane was also conducted.

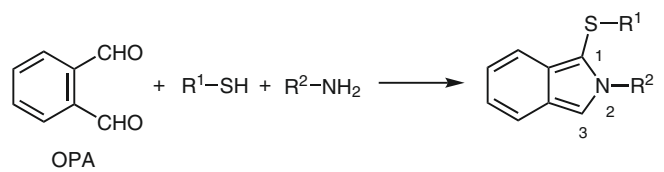

Scheme 1 The reaction of OPA with a primary amine and thiol

A novel series of 1-alkylthio-2-alkyl-substituted isoindoles, which are stable enough to be isolated, has been designed and synthesized, making use of $C_{3}$-symmetric primary amines in the OPA method. The $C_{3}$-symmetric primary amines employed in this study were chosen to enhance the steric protection effect in isoindole molecules by further increasing the steric bulk of the tert-butylamine. Thus, a series of $C_{3}$-symmetric primary amines $\mathbf{5 a} \mathbf{a}-\mathbf{g}$ was prepared from tris(hydroxymethyl)aminomethane (1) or tris(hydroxypropyl)aminomethane (2), as shown in Scheme 

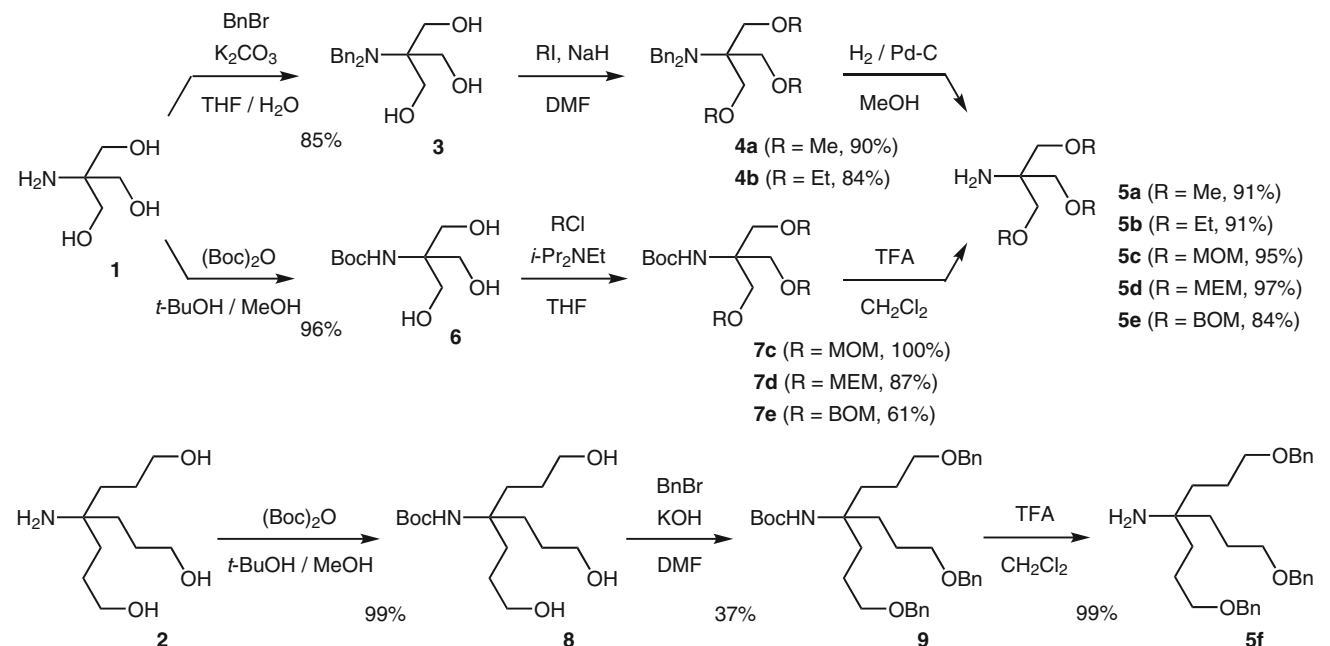

2

8

9

$5 f$

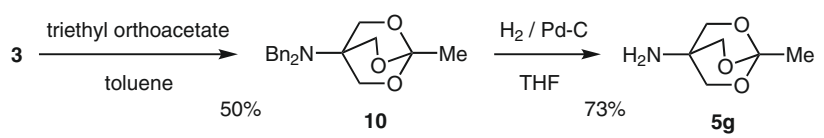

Scheme 2 Synthesis of $C_{3}$-symmetric primary amines $\mathbf{5 a - g}$ starting from tris(hydroxymethyl)aminomethane (1) and tris(hydroxypropyl)aminomethane (2)

2. N,N-Dibenzyl tris(hydroxymethyl)aminomethane (3) was synthesized from $\mathbf{1}$ using benzyl bromide according to a modified procedure. ${ }^{9}$ O-Alkylation of $\mathbf{3}$ with alkyl iodides followed by hydrogenolysis catalyzed by palladium on activated charcoal (Pd-C) gave primary amines $\mathbf{5 a}$ and $\mathbf{5 b}$, respectively. $\mathrm{N}$-Boc-tris(hydroxymethyl)aminomethane $(\mathbf{6}),{ }^{10}$ obtained from $\mathbf{1}$, was $\mathrm{O}$-alkylated by the corresponding alkyl chlorides to afford $N$-Boc-protected amines $7 c-e$. Deprotection of 7c-e using trifluoroacetic acid (TFA) gave primary amines $\mathbf{5 c}-\mathbf{e}$. $\mathrm{O}$-Benzylated amine $\mathbf{5 f}$ was also synthesized from 2 according to a similar procedure used for the preparation of $\mathbf{5 c}-\mathbf{e}$. In addition, reaction of $\mathbf{3}$ with triethyl orthoacetate followed by removal of the benzyl groups by hydrogenolysis furnished $\mathbf{5 g},{ }^{11}$ which was regarded as a less bulky primary amine than the other 0 -protected amines $\mathbf{5 a}-\mathbf{f}$.

In our attempt to synthesize stable and isolable 1-alkylthio-2-alkyl-substituted isoindoles by the OPA method, $C_{3}$-symmetric primary amines $\mathbf{1}, \mathbf{2}$, and $\mathbf{5 a}$-e were reacted with OPA and 1-propanethiol in anhydrous $\mathrm{MeOH}$ at room temperature in the dark using brown-tinted glassware as shown in Table 1. 0 -Protected amines $\mathbf{5 a}, \mathbf{b}$ afforded isoindoles 11a,b with small amounts of isoindolin-1-ones 12a,b as by-products (entries 1 and 2). As expected, isoindoles 11a,b were stable and isolable by column chromatography on silica gel. When amines $\mathbf{5 c}-\mathbf{e}$ were employed, the isolated yields of isoindoles 11c-e (54-60\%) were relatively low, and isoindolin-1-ones 12c-e were obtained in 39-44\% yields (entries $3-5$ ). To examine the influence of the oxygen atom of $C_{3}$-symmetric amine 5e originating from $\mathbf{1}$ on the formation of isoindolin-1-one 12e, $O$-benzylated
Table 1 Synthesis of 1-Alkylthio-2-alkyl-Substituted Isoindoles 11a-i by the OPA Method ${ }^{a}$
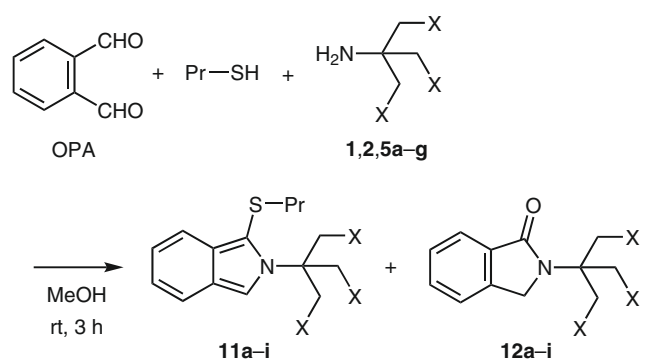

\begin{tabular}{|c|c|c|c|}
\hline Entry & $x$ & Yield of $11(\%)^{b}$ & Yield of $12(\%)^{b}$ \\
\hline 1 & OMe (5a) & 65 (11a) & $18(\mathbf{1 2 a})$ \\
\hline 2 & OEt (5b) & 85 (11b) & 12 (12b) \\
\hline 3 & OMOM (5c) & $57(\mathbf{1 1 c})$ & $41(\mathbf{1 2 c})$ \\
\hline 4 & OMEM (5d) & 54 (11d) & 44 (12d) \\
\hline 5 & OBOM (5e) & 60 (11e) & $39(\mathbf{1 2 e})$ \\
\hline 6 & $\left(\mathrm{CH}_{2}\right)_{2} \mathrm{OBn}(\mathbf{5 f})$ & $93(\mathbf{1 1 f})$ & ca. $6(\mathbf{1 2 f})^{c}$ \\
\hline 7 & orthoester $\mathbf{( 5 g}$ ) & $-^{d}(\mathbf{1 1 g})$ & $0(\mathbf{1 2 g})$ \\
\hline 8 & $\mathrm{OH}(\mathbf{1})$ & $-^{d}(11 \mathrm{~h})$ & $0(\mathbf{1 2 h})$ \\
\hline 9 & $\left(\mathrm{CH}_{2}\right)_{2} \mathrm{OH}(\mathbf{2})$ & $90(\mathbf{1 1 i})$ & $0(\mathbf{1 2 i})$ \\
\hline $10^{\mathrm{e}}$ & OMOM (5c) & $-(11 c)$ & $56(12 c)$ \\
\hline $11^{\mathrm{e}}$ & $\left(\mathrm{CH}_{2}\right)_{2} \mathrm{OH}(\mathbf{2})$ & $-(11 i)$ & $0(\mathbf{1 2 i})$ \\
\hline
\end{tabular}

a Reaction conditions: OPA (1 equiv), 1-propanethiol (1.1 equiv), amine (1.1 equiv).

${ }^{\mathrm{b}}$ Isolated yield.

' Small amounts of impurities were included.

d Too labile to be isolated.

e Reaction without 1-propanethiol. 
tris(hydroxypropyl)aminomethane $\mathbf{5 f}$ was reacted with OPA and 1-propanethiol. As a result, $\mathbf{5 f}$ afforded isoindole 11f in 93\% yield and isoindolin-1-one $\mathbf{1 2 f}$ in ca. 6\% yield (entry 6). On the other hand, isoindole 11g, synthesized from less bulky amine $\mathbf{5 g}$, was too labile to be isolated (entry 7). In the reaction of triols $\mathbf{1}$ and $\mathbf{2}$, the 1-alkylthio-2alkyl-substituted isoindoles $11 \mathrm{~h}$ and $11 \mathbf{i}$ were also obtained, but $\mathbf{1 1 h}$ decomposed immediately after isolation by column chromatography on silica gel (entries 8 and 9). In addition, the reaction of OPA with amine $\mathbf{5 c}$ in the absence of 1-propanethiol was relatively slow and afforded isoindolin-1-one $\mathbf{1 2 c}$ in $56 \%$ yield (entry 10). In the reaction of $\mathbf{2}$ and OPA without 1-propanethiol, isoindolin-1-one 12i was not obtained at all (entry 11). When isoindole 11c, instead of OPA, was placed under the same reaction conditions as those noted in entry 3, 86\% yield of 11c was recovered without production of 12c. Thus, the formation of isoindolin-1-one $12 \mathrm{c}$ is presumed not to be attributable to the hydrolysis of 11c under these reaction conditions.

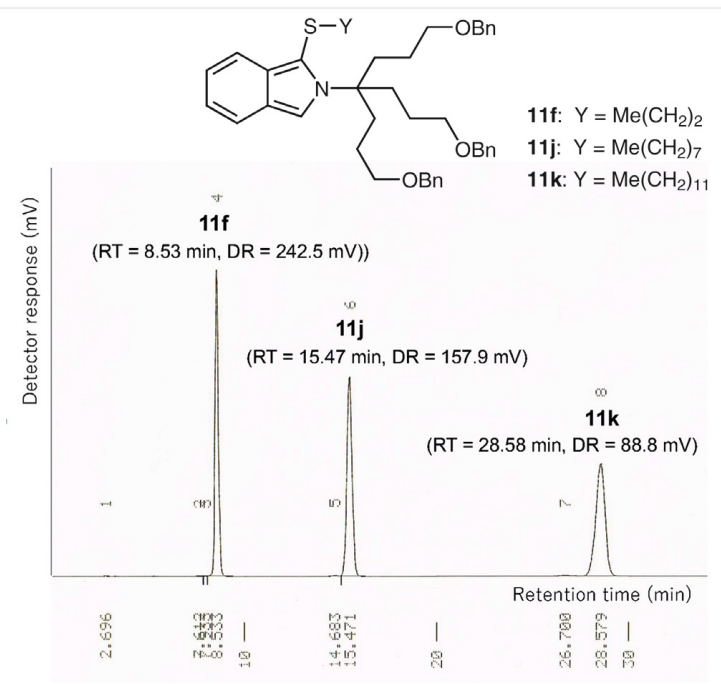

Figure $1 \mathrm{HPLC}$ analysis for resolution of a mixture of isoindoles $\mathbf{1 1 f}$, $\mathbf{1 1} \mathbf{j}$, and $\mathbf{1 1} \mathbf{k}$ formed from 1-propanethiol, 1-octanethiol, and 1-dodecanethiol, respectively
Based on these results, plausible reaction pathways for the formation of isoindoles 11a-e (route A) and isoindolin1-ones 12a-e (route $B$ ) have been proposed (Scheme 3 ). Route $\mathrm{A}$ is the standard pathway for the three-component coupling reaction based on the OPA method. ${ }^{5}$ On the other hand, neighboring group participation by an oxygen atom of the ether bond of each of amines $\mathbf{5 a}-\mathbf{e}$ is involved in route $\mathrm{B}$. In this case, the formation of the five-membered 1,3-oxazolidin-1-ium intermediate by intramolecular cyclization prevents intermolecular nucleophilic attack of 1propanethiol. The ratios of the products of 11a-e and 12ae seem to depend on the bond angle $\theta(\mathrm{N}-\mathrm{C}-\mathrm{C})$ of the corresponding $C_{3}$-symmetric primary amines $\mathbf{5 a}-\mathbf{e}$. Amines $\mathbf{5 c}$ e, which are presumed to have smaller $\theta$ values compared with $\mathbf{5 a}$ and $\mathbf{5 b}$, are liable to follow pathway B. In the case of amine $\mathbf{5 f}$, in which three oxygen atoms of $\mathbf{5 e}$ are replaced by three methylene units, this kind of neighboring group participation of an oxygen atom via a seven-membered 1,3oxazepan-1-ium intermediate might be difficult to achieve.

Furthermore, 1-octanethiol and 1-dodecanethiol readily reacted with OPA and $\mathbf{5 f}$ to afford stable and isolable isoindoles $\mathbf{1 1} \mathbf{j}$ and $11 \mathbf{k}$ in $96 \%$ and $91 \%$ yields, respectively. To provide some preliminary data on the application as a novel method of analyzing thiols, HPLC analysis of an equimolar mixture of isoindoles $\mathbf{1 1 f}, \mathbf{1 1} \mathbf{j}$, and 11k was investigated with an ODS column. The results showed that isoindoles $\mathbf{1 1 f}, \mathbf{1 1} \mathbf{j}$, and $11 \mathbf{k}$ were easily resolved from one another, as shown in Figure 1. The area ratio for these three peaks derived from isoindoles 11f, 11j, and 11k (32.35:33.94:33.18) was almost identical to the respective molar ratio (1:1:1).

In summary, we have synthesized a novel series of 1 -alkylthio-2-alkyl-substituted isoindoles 11a-f and 11i-k by the three-component coupling reaction of OPA, thiols (1propanethiol, 1-octanethiol, and 1-dodecanthiol), and $C_{3}$ symmetric bulky amines $\mathbf{2}$ and $\mathbf{5 a}-\mathbf{f}$. These isoindoles were stable enough to be isolated by column chromatography over silica gel. To the best of our knowledge, this is the first report of the synthesis of stable and isolable isoindoles based on the OPA method using modest molecular weight amines. Efforts toward the development of a novel analytical

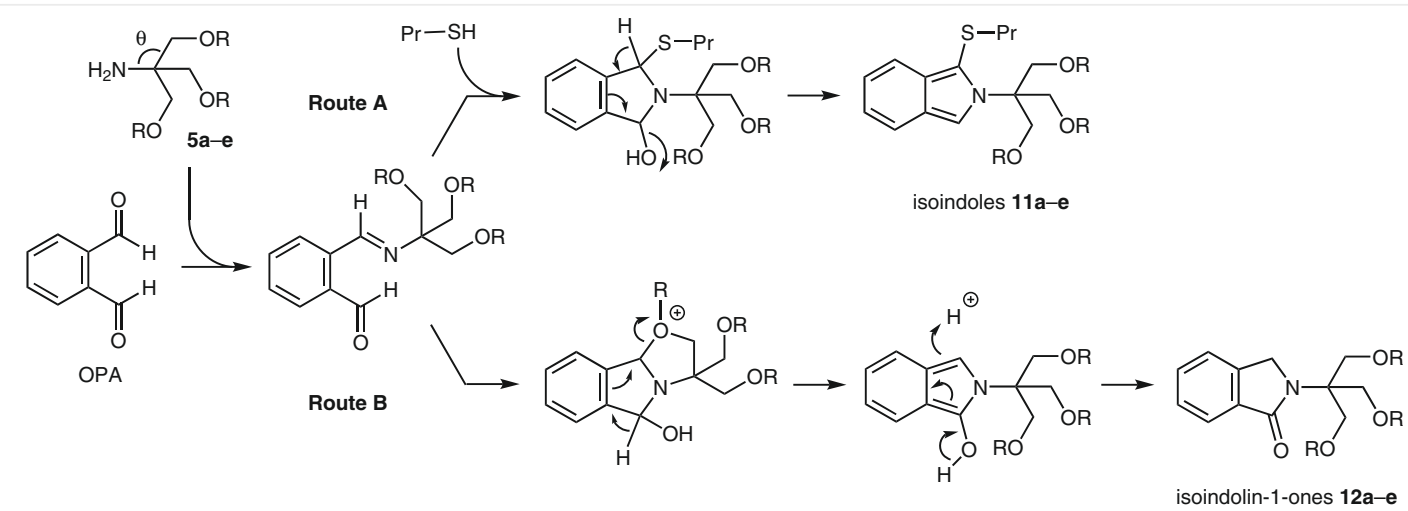

Scheme 3 Plausible reaction pathways for the formation of 11a-e and 12a-e 
method for thiols by employing the formation of sterically protected isoindoles based on the OPA method are under way.

All melting points were determined with a Yanagimoto micro melting point apparatus and are uncorrected. IR spectra were obtained with a JASCO FT/IR-6200 IR Fourier transform spectrometer. ${ }^{1} \mathrm{H}$ NMR (500 $\mathrm{MHz}$ ) and ${ }^{13} \mathrm{C}$ NMR (125 MHz) spectra were recorded with a Bruker AV500 spectrometer. Chemical shifts are given in $\delta$ values (ppm) using TMS as an internal standard. HRMS (ESI) were recorded with a Waters LCT Premier spectrometer. Elemental combustion analyses were performed with a J-SCIENCE LAB JM10 microcorder. All reactions were monitored by TLC employing $0.25 \mathrm{~mm}$ silica gel plates (Merck 5715; $60 \mathrm{~F}_{254}$ ). Column chromatography was carried out on silica gel [Silica Gel 60N (Kanto Chemical) or Silica Gel PSQ 60B (Fuji Silysia Chemical)]. Anhydrous THF, $\mathrm{CH}_{2} \mathrm{Cl}_{2}$, DMF, and toluene were used as purchased from Kanto Chemical. All other reagents were used as purchased.

\section{$\mathrm{N}, \mathrm{N}$-Dibenzyl-1,3-dimethoxy-2-(methoxymethyl)propan-2-amine (4a)}

To a suspension of $\mathrm{NaH}$ (50-72\% in mineral oil, $394 \mathrm{mg}, 8.21-11.8$ $\mathrm{mmol}$ ) in anhydrous DMF (5 mL) was added 3 (900 $\mathrm{mg}, 2.99 \mathrm{mmol})$ in anhydrous DMF $(5 \mathrm{~mL})$ and MeI $(614 \mu \mathrm{L}, 9.85 \mathrm{mmol})$. The reaction mixture was stirred at r.t. for $4 \mathrm{~h}$ under argon. $\mathrm{H}_{2} \mathrm{O}(10 \mathrm{~mL})$ was added and the reaction mixture was extracted with EtOAc $(50 \mathrm{~mL})$. The organic layer was washed with $\mathrm{H}_{2} \mathrm{O}(3 \times 20 \mathrm{~mL})$, dried $\left(\mathrm{MgSO}_{4}\right)$, filtered, and concentrated in vacuo. The oily residue was purified by column chromatography [silica gel 60N; $n$-hexane-EtOAc, 9:1] to afford $4 a$.

Yield: $922 \mathrm{mg}$ (90\%); pale-yellow oil.

IR (neat): 3026, 2980, 2889, 2810, 2059, 1946, 1869, 1810, 1603, $1453,1107 \mathrm{~cm}^{-1}$.

${ }^{1} \mathrm{H}$ NMR $\left(500 \mathrm{MHz}, \mathrm{CDCl}_{3}\right): \delta=7.27-7.26(\mathrm{~m}, 4 \mathrm{H}), 7.16-7.13(\mathrm{~m}, 4 \mathrm{H})$, 7.08-7.05 (m, $2 \mathrm{H}), 3.98$ (s, $4 \mathrm{H}), 3.52(\mathrm{~s}, 6 \mathrm{H}), 3.25(\mathrm{~s}, 9 \mathrm{H})$.

${ }^{13} \mathrm{C}$ NMR $\left(125 \mathrm{MHz}, \mathrm{CDCl}_{3}\right): \delta=142.4,128.4,127.6,126.0,73.6,64.0$, $59.1,54.8$.

HRMS (ESI): $m / z[\mathrm{M}+\mathrm{Na}]^{+}$calcd for $\mathrm{C}_{21} \mathrm{H}_{29} \mathrm{NO}_{3} \mathrm{Na}$ : 366.2045; found: 366.2031.

Anal. Calcd for $\mathrm{C}_{21} \mathrm{H}_{29} \mathrm{NO}_{3}$ : C, 73.44; $\mathrm{H}, 8.51 ; \mathrm{N}, 4.08$. Found: C, 73.15; H, 8.65; N, 4.09.

\section{$N, N$-Dibenzyl-1,3-diethoxy-2-(ethoxymethyl)propan-2-amine (4b)}

Synthesized from $\mathbf{3}$ and ethyl iodide according to the procedure used to prepare $\mathbf{4 a}$.

Yield: $467 \mathrm{mg}$ (84\%); pale-yellow oil.

IR (neat): 2974, 2867, 1494, 1454, 1377, $1113 \mathrm{~cm}^{-1}$.

${ }^{1} \mathrm{H} \mathrm{NMR}\left(500 \mathrm{MHz}, \mathrm{CDCl}_{3}\right): \delta=7.29-7.26(\mathrm{~m}, 4 \mathrm{H}), 7.15-7.12(\mathrm{~m}, 4 \mathrm{H})$, 7.07-7.04 (m, $2 \mathrm{H}), 4.00$ (s, $4 \mathrm{H}), 3.55(\mathrm{~s}, 6 \mathrm{H}), 3.38$ (q, J = 7.0 Hz, $6 \mathrm{H})$, $1.16(\mathrm{t}, J=7.0 \mathrm{~Hz}, 9 \mathrm{H})$.

${ }^{13} \mathrm{C}$ NMR $\left(125 \mathrm{MHz}, \mathrm{CDCl}_{3}\right): \delta=142.8,128.4,127.5,125.8,71.3,66.6$, $64.1,54.8,15.2$.

HRMS (ESI): $m / z$ [M $+\mathrm{Na}]^{+}$calcd for $\mathrm{C}_{24} \mathrm{H}_{35} \mathrm{NO}_{3} \mathrm{Na}$ : 408.2515; found: 408.2504.

Anal. Calcd for $\mathrm{C}_{24} \mathrm{H}_{35} \mathrm{NO}_{3}$ : C, 74.77; $\mathrm{H}, 9.15 ; \mathrm{N}, 3.63$. Found: $\mathrm{C}, 74.62$; $\mathrm{H}, 9.31 ; \mathrm{N}, 3.58$.

\section{1,3-Dimethoxy-2-(methoxymethyl)propan-2-amine (5a)}

A mixture of $4 \mathbf{a}(270 \mathrm{mg}, 0.790 \mathrm{mmol})$ and $10 \% \mathrm{Pd} / \mathrm{C}(84.0 \mathrm{mg}, 0.0790$ $\mathrm{mmol})$ in $\mathrm{MeOH}(10 \mathrm{~mL})$ was stirred at r.t. for $1.5 \mathrm{~h}$ under $\mathrm{H}_{2}$ atmosphere. The reaction mixture was filtered and concentrated in vacuo. The residue was dissolved in $\mathrm{CHCl}_{3}(30 \mathrm{~mL})$ and washed with sat. aq $\mathrm{NaHCO}_{3}(20 \mathrm{~mL})$. The organic layer was dried $\left(\mathrm{MgSO}_{4}\right)$, filtered, and concentrated in vacuo to afford $\mathbf{5 a}$.

Yield: $118 \mathrm{mg}$ (91\%); pale-yellow oil. IR (neat): 3383, 2980, 2892, 2814, 1456, $1109 \mathrm{~cm}^{-1}$. ${ }^{1} \mathrm{H}$ NMR (500 MHz, $\mathrm{CDCl}_{3}$ ): $\delta$ = $3.35(\mathrm{~s}, 9 \mathrm{H}), 3.29$ (s, $\left.6 \mathrm{H}\right), 1.52$ (br s, $2 \mathrm{H})$.

${ }^{13} \mathrm{C}$ NMR $\left(125 \mathrm{MHz}, \mathrm{CDCl}_{3}\right): \delta=75.0,59.4,55.7$.

HRMS (ESI): $m / z$ [M $+\mathrm{Na}]^{+}$calcd for $\mathrm{C}_{7} \mathrm{H}_{17} \mathrm{NO}_{3} \mathrm{Na}$ : 186.1106; found: 186.1091 .

\section{1,3-Diethoxy-2-(ethoxymethyl)propan-2-amine (5b)}

Synthesized from $\mathbf{4 b}$ according to the procedure used to prepare $\mathbf{5 a}$. Yield: $170 \mathrm{mg}$ (91\%); pale-yellow oil.

IR (neat): 3385, 2976, 2867, 1110, $864 \mathrm{~cm}^{-1}$.

${ }^{1} \mathrm{H} \mathrm{NMR}\left(500 \mathrm{MHz}, \mathrm{CDCl}_{3}\right): \delta=3.49(\mathrm{q}, J=7.0 \mathrm{~Hz}, 6 \mathrm{H}), 3.33(\mathrm{~s}, 6 \mathrm{H})$, 1.55 (br s, $2 \mathrm{H}), 1.17(\mathrm{t}, J=7.0 \mathrm{~Hz}, 9 \mathrm{H})$.

${ }^{13} \mathrm{C}$ NMR $\left(125 \mathrm{MHz}, \mathrm{CDCl}_{3}\right): \delta=72.6,66.8,55.7,15.1$.

HRMS (ESI): $m / z[\mathrm{M}+\mathrm{Na}]^{+} \mathrm{C}_{10} \mathrm{H}_{23} \mathrm{NO}_{3} \mathrm{Na}$ : 228.1576; found: 228.1565 .

\section{tert-Butyl [1,3-Dihydroxy-2-(hydroxymethyl)propan-2-yl]carba-} mate (6) $)^{10 \mathrm{~b}}$

To a solution of $1(2.00 \mathrm{~g}, 16.5 \mathrm{mmol})$ in $t-\mathrm{BuOH}(27.5 \mathrm{~mL}) / \mathrm{MeOH}$ $(12.5 \mathrm{~mL})$ was added di-tert-butyl dicarbonate $(4.70 \mathrm{~g}, 21.5 \mathrm{mmol})$. After stirring for $18 \mathrm{~h}$ at r.t., the reaction mixture was evaporated in vacuo and the residue was washed with cool EtOAc to afford 6.

Yield: $3.52 \mathrm{~g}$ (96\%); white solid; $\mathrm{mp} 142.7-145^{\circ} \mathrm{C}$ (colorless needles, EtOAc).

IR (KBr): 3300, 2962, 1680, 1549, 1468, 1369, 1296, $1176 \mathrm{~cm}^{-1}$.

${ }^{1} \mathrm{H}$ NMR $\left(500 \mathrm{MHz}\right.$, DMSO- $\left.d_{6}\right): \delta=5.76(\mathrm{br} \mathrm{s}, 1 \mathrm{H}), 4.50(\mathrm{t}, J=5.3 \mathrm{~Hz}$, $3 \mathrm{H}), 3.51$ (d, $J=5.6 \mathrm{~Hz}, 6 \mathrm{H}), 1.37(\mathrm{~s}, 9 \mathrm{H})$.

${ }^{13} \mathrm{C}$ NMR (125 MHz, DMSO- $d_{6}$ ): $\delta=154.9,77.7,60.3,60.1,28.1$.

HRMS (ESI): $m / z[\mathrm{M}+\mathrm{Na}]^{+}$calcd for $\mathrm{C}_{9} \mathrm{H}_{19} \mathrm{NO}_{5} \mathrm{Na}$ : 244.1161; found: 244.1141.

Anal. Calcd for $\mathrm{C}_{9} \mathrm{H}_{19} \mathrm{NO}_{5}$ : C, 48.86; $\mathrm{H}, 8.66 ; \mathrm{N}, 6.33$. Found: $\mathrm{C}, 48.73$; $\mathrm{H}, 8.68 ; \mathrm{N}, 6.31$.

tert-Butyl \{6-[(Methoxymethoxy)methyl]-2,4,8,10-tetraoxaundecan-6-yl\}carbamate (7c)

To a solution of $6(500 \mathrm{mg}, 2.26 \mathrm{mmol})$ and DIPEA $(1.30 \mathrm{~mL}, 7.46$ $\mathrm{mmol})$ in anhydrous THF $(6.6 \mathrm{~mL})$ was added $\mathrm{MOMCl}(0.790 \mathrm{~mL}, 7.46$ $\mathrm{mmol})$. After stirring for $2 \mathrm{~h}$ at r.t., DIPEA $(1.30 \mathrm{~mL}, 7.46 \mathrm{mmol})$ and MOMCl $(0.790 \mathrm{~mL}, 7.46 \mathrm{mmol})$ were added to the reaction mixture. After stirring for a further $2 \mathrm{~h}$ at r.t., DIPEA $(1.30 \mathrm{~mL}, 7.46 \mathrm{mmol})$ and $\mathrm{MOMCl}(0.790 \mathrm{~mL}, 7.46 \mathrm{mmol})$ were again added to the reaction mixture. After stirring for a final $2 \mathrm{~h}$ at r.t., the reaction mixture was evaporated in vacuo. The residue was dissolved in EtOAc $(50 \mathrm{~mL})$ and washed with brine $(2 \times 20 \mathrm{~mL})$. The organic layer was dried $\left(\mathrm{Na}_{2} \mathrm{SO}_{4}\right)$, filtered, and concentrated in vacuo. The oily residue was purified by column chromatography [silica gel PSQ 60B; $n$-hexane-EtOAc, 2:1] to afford 7c.

Yield: 796 mg (100\%); pale-yellow oil. 
IR (neat): 3450, 3359, 2934, 2889, 2824, 1718, 1502, 1366, 1245 , $1150,1112,1047 \mathrm{~cm}^{-1}$.

${ }^{1} \mathrm{H} \mathrm{NMR}\left(500 \mathrm{MHz}, \mathrm{CDCl}_{3}\right): \delta=5.03(\mathrm{br} \mathrm{s}, 1 \mathrm{H}), 4.63(\mathrm{~s}, 6 \mathrm{H}), 3.82(\mathrm{~s}$, $6 \mathrm{H}), 3.36(\mathrm{~s}, 9 \mathrm{H}), 1.42(\mathrm{~s}, 9 \mathrm{H})$.

${ }^{13} \mathrm{C} \mathrm{NMR}\left(125 \mathrm{MHz}, \mathrm{CDCl}_{3}\right): \delta=154.7,96.8,79.1,67.1,57.8,55.3,28.3$. HRMS (ESI): $m / z[\mathrm{M}+\mathrm{Na}]^{+}$calcd for $\mathrm{C}_{15} \mathrm{H}_{31} \mathrm{NO}_{8} \mathrm{Na}$ : 376.1947 ; found: 376.1922.

Anal. Calcd for $\mathrm{C}_{15} \mathrm{H}_{31} \mathrm{NO}_{8}$ : C, 50.98; $\mathrm{H}, 8.84 ; \mathrm{N}, 3.96$. Found: $\mathrm{C}, 50.68$; $\mathrm{H}, 8.83 ; \mathrm{N}, 4.02$.

\section{tert-Butyl (9-\{[(2-Methoxyethoxy)methoxy]methyl\}-}

2,5,7,11,13,16-hexaoxaheptadecan-9-yl)carbamate (7d)

Synthesized from 6 and $\mathrm{MEMCl}$ according to the procedure used to prepare 7c.

Yield: $576 \mathrm{mg}$ (87\%); pale-yellow oil.

IR (neat): 3450, 3348, 2931, 2885, 1716, 1500, 1456, 1366, 1246, $1173,1116,1045 \mathrm{~cm}^{-1}$.

${ }^{1} \mathrm{H} \mathrm{NMR}\left(500 \mathrm{MHz}, \mathrm{CDCl}_{3}\right): \delta=5.08(\mathrm{br} \mathrm{s}, 1 \mathrm{H}), 4.71(\mathrm{~s}, 6 \mathrm{H}), 3.82(\mathrm{~s}$, $6 \mathrm{H}), 3.69-3.67$ (m, $6 \mathrm{H}), 3.56-3.54(\mathrm{~m}, 6 \mathrm{H}), 3.39(\mathrm{~s}, 9 \mathrm{H}), 1.41(\mathrm{~s}, 9 \mathrm{H})$.

${ }^{13} \mathrm{C}$ NMR $\left(125 \mathrm{MHz}, \mathrm{CDCl}_{3}\right): \delta=154.7,95.9,79.1,71.7,67.3,66.9,59.0$, 57.9, 28.3.

HRMS (ESI): $m / z[\mathrm{M}+\mathrm{Na}]^{+}$calcd for $\mathrm{C}_{21} \mathrm{H}_{43} \mathrm{NO}_{11} \mathrm{Na}$ : 508.2734 ; found: 508.2727 .

\section{tert-Butyl (6-\{[(Benzyloxy)methoxy]methyl $\}-1,11-d i p h e n y l-$} 2,4,8,10-tetraoxaundecan-6-yl)carbamate (7e) ${ }^{10 \mathrm{~b}}$

Synthesized from $\mathbf{6}$ and $\mathrm{BOMCl}$ according to the procedure used to prepare $\mathbf{7 c}$.

Yield: $481 \mathrm{mg}$ (61\%); pale-yellow oil.

IR (neat): 3436, 3063, 3032, 2939, 2885, 1716, 1498, 1366, 1170, $1112,1046 \mathrm{~cm}^{-1}$.

${ }^{1} \mathrm{H}$ NMR (500 MHz, $\left.\mathrm{CDCl}_{3}\right): \delta=7.34-7.27(\mathrm{~m}, 15 \mathrm{H}$ ), 5.01 (br s, $1 \mathrm{H}$ ), 4.76 (s, $6 \mathrm{H}), 4.59$ (s, $6 \mathrm{H}), 3.91(\mathrm{~s}, 6 \mathrm{H}), 1.41(\mathrm{~s}, 9 \mathrm{H})$.

${ }^{13} \mathrm{C}$ NMR $\left(125 \mathrm{MHz}, \mathrm{CDCl}_{3}\right): \delta=154.7,137.7,128.4,127.9,127.7,95.0$, $79.2,69.4,67.4,57.9,28.4$.

HRMS (ESI): $m / z[\mathrm{M}+\mathrm{Na}]^{+}$calcd for $\mathrm{C}_{33} \mathrm{H}_{43} \mathrm{NO}_{8} \mathrm{Na}$ : 604.2886; found: 604.2878 .

\section{6-[(Methoxymethoxy)methyl]-2,4,8,10-tetraoxaundecan-6-amine} (5c)

To a solution of $7 \mathbf{c}(400 \mathrm{mg}, 1.13 \mathrm{mmol})$ in anhydrous $\mathrm{CH}_{2} \mathrm{Cl}_{2}(5.5 \mathrm{~mL})$ was added TFA $(2.00 \mathrm{~mL}, 26.0 \mathrm{mmol})$ at $0{ }^{\circ} \mathrm{C}$. After stirring for $50 \mathrm{~min}$ at $0{ }^{\circ} \mathrm{C}, 2 \mathrm{~N} \mathrm{Na}_{2} \mathrm{CO}_{3}(25 \mathrm{~mL})$ was added and the mixture was extracted with $\mathrm{CHCl}_{3}(3 \times 30 \mathrm{~mL})$. The combined organic layers were dried $(\mathrm{Mg}-$ $\mathrm{SO}_{4}$ ), filtered, and concentrated in vacuo. The oily residue was purified by column chromatography [silica gel PSQ 60B; $\mathrm{CHCl}_{3}-\mathrm{MeOH}$, 9:1] to afford $\mathbf{5 c}$.

Yield: $272 \mathrm{mg}$ (95\%); pale-yellow oil.

IR (neat): 3378, 2929, 2885, 1587, 1467, 1442, 1403, 1213, 1149, $1110,1040 \mathrm{~cm}^{-1}$.

${ }^{1} \mathrm{H} \mathrm{NMR}\left(500 \mathrm{MHz}, \mathrm{CDCl}_{3}\right): \delta=4.64(\mathrm{~s}, 6 \mathrm{H}), 3.49(\mathrm{~s}, 6 \mathrm{H}), 3.36(\mathrm{~s}, 9 \mathrm{H})$, 1.56 (br s, $2 \mathrm{H}$ ).

${ }^{13} \mathrm{C} \mathrm{NMR}\left(125 \mathrm{MHz}, \mathrm{CDCl}_{3}\right): \delta=96.8,69.9,55.3,55.2$.

HRMS (ESI): $m / z[\mathrm{M}+\mathrm{Na}]^{+}$calcd for $\mathrm{C}_{10} \mathrm{H}_{23} \mathrm{NO}_{6} \mathrm{Na}$ : 276.1423; found: 276.1401 .
9-\{[(2-Methoxyethoxy)methoxy]methyl\}-2,5,7,11,13,16-hexaoxaheptadecan-9-amine (5d)

Synthesized from $\mathbf{7 d}$ according to the procedure used to prepare $\mathbf{5 c}$. Yield: $193 \mathrm{mg}$ (97\%); colorless oil.

IR (neat): 2928, 2881, 1457, 1413, 1365, 1244, 1200, 1117, $1043 \mathrm{~cm}^{-1}$. ${ }^{1} \mathrm{H}$ NMR $\left(500 \mathrm{MHz}, \mathrm{CDCl}_{3}\right): \delta=4.72(\mathrm{~s}, 6 \mathrm{H}), 3.69-3.67(\mathrm{~m}, 6 \mathrm{H}), 3.56-$ $3.55(\mathrm{~m}, 6 \mathrm{H}), 3.50(\mathrm{~s}, 6 \mathrm{H}), 3.40(\mathrm{~s}, 9 \mathrm{H}), 1.66(\mathrm{br} \mathrm{s}, 2 \mathrm{H})$.

${ }^{13} \mathrm{C}$ NMR $\left(125 \mathrm{MHz}, \mathrm{CDCl}_{3}\right): \delta=95.9,71.7,70.1,66.8,59.0,55.4$. HRMS (ESI): $m / z[\mathrm{M}+\mathrm{Na}]^{+}$calcd for $\mathrm{C}_{16} \mathrm{H}_{35} \mathrm{NO}_{9} \mathrm{Na}$ : 408.2210; found: 408.2204.

6-\{[(Benzyloxy)methoxy]methyl $\}-1,11-d i p h e n y l-2,4,8,10-$ tetraoxaundecan-6-amine (5e) $)^{10 \mathrm{~b}}$

Synthesized from $\mathbf{7 e}$ according to the procedure used to prepare $\mathbf{5 c}$.

Yield: $140 \mathrm{mg}(84 \%)$; pale-yellow oil.

IR (neat): 3379, 3088, 3063, 3031, 2936, 2880, 1586, 1498, 1455, $1381,1162,1111,1041 \mathrm{~cm}^{-1}$.

${ }^{1} \mathrm{H}$ NMR $\left(500 \mathrm{MHz}, \mathrm{CDCl}_{3}\right): \delta=7.36-7.27(\mathrm{~m}, 15 \mathrm{H}), 4.77(\mathrm{~s}, 6 \mathrm{H}), 4.59$ (s, $6 \mathrm{H}), 3.58$ (s, $6 \mathrm{H}), 1.62$ (br s, $2 \mathrm{H})$.

${ }^{13} \mathrm{C}$ NMR $\left(125 \mathrm{MHz}, \mathrm{CDCl}_{3}\right): \delta=137.7,128.4,127.8,127.7,95.0,70.2$, 69.4, 55.4.

HRMS (ESI): $m / z[\mathrm{M}+\mathrm{Na}]^{+}$calcd for $\mathrm{C}_{28} \mathrm{H}_{35} \mathrm{NO}_{6} \mathrm{Na}$ : 504.2362 ; found: 504.2313 .

tert-Butyl [1,7-Dihydroxy-4-(3-hydroxypropyl)heptan-4-yl]carbamate (8)

Synthesized from $\mathbf{2}$ according to the procedure used to prepare $\mathbf{6}$.

Yield: $1.62 \mathrm{~g}$ (99\%); mp 103.7-105 ${ }^{\circ} \mathrm{C}$ (colorless needles, $\mathrm{CHCl}_{3}$ ).

IR (KBr): 3405, 3305, 3262, 2949, 2873, 1691, 1546, 1454, 1366, 1279 , $1166 \mathrm{~cm}^{-1}$.

${ }^{1} \mathrm{H}$ NMR $\left(500 \mathrm{MHz}\right.$, DMSO- $\left.d_{6}\right): \delta=6.07$ (br s, $\left.1 \mathrm{H}\right), 4.36(\mathrm{t}, J=5.0 \mathrm{~Hz}$, $3 \mathrm{H}), 3.34-3.31$ (m, $6 \mathrm{H}), 1.49-1.47(\mathrm{~m}, 6 \mathrm{H}), 1.36$ (s, $9 \mathrm{H}), 1.36-1.27$ ( $\mathrm{m}, 6 \mathrm{H})$.

${ }^{13} \mathrm{C}$ NMR $\left(125 \mathrm{MHz}\right.$, DMSO- $\left.d_{6}\right): \delta=153.9,76.7,61.3,56.1,31.0,28.2$, 26.5 .

HRMS (ESI): $m / z[\mathrm{M}+\mathrm{Na}]^{+}$calcd for $\mathrm{C}_{15} \mathrm{H}_{31} \mathrm{NO}_{5} \mathrm{Na}$ : 328.2100; found: 328.2104.

Anal. Calcd for $\mathrm{C}_{15} \mathrm{H}_{31} \mathrm{NO}_{5}$ : C, 58.99; $\mathrm{H}, 10.23 ; \mathrm{N}, 4.59$. Found: $\mathrm{C}, 58.69$; $\mathrm{H}, 10.16 ; \mathrm{N}, 4.53$.

tert-Butyl \{1,7-Bis(benzyloxy)-4-[3-(benzyloxy)propyl]heptan-4yl\}carbamate (9)

To a solution of 8 (300 mg, $0.982 \mathrm{mmol})$ and benzyl bromide $(0.760$ $\mathrm{mL}, 6.38 \mathrm{mmol})$ in anhydrous DMF (1.6 mL) was added $\mathrm{KOH}$ (379 mg, $6.75 \mathrm{mmol}$ ) at r.t. under argon. The reaction mixture was stirred for $21 \mathrm{~h}$ at r.t., for $2 \mathrm{~h}$ at $40{ }^{\circ} \mathrm{C}$, and for $2 \mathrm{~h}$ at $60^{\circ} \mathrm{C} . \mathrm{H}_{2} \mathrm{O}(20 \mathrm{~mL})$ was added and the mixture was extracted with EtOAc $(30 \mathrm{~mL})$. The organic layer was washed with $\mathrm{H}_{2} \mathrm{O}(2 \times 20 \mathrm{~mL})$, dried $\left(\mathrm{Na}_{2} \mathrm{SO}_{4}\right)$, filtered, and concentrated in vacuo. The oily residue was purified by column chromatography [silica gel PSQ 60B; $n$-hexane-EtOAc, 5:1 to 1:1] to afford 9.

Yield: $211 \mathrm{mg}$ (37\%); colorless oil.

IR (neat): 3352, 2945, 2856, 1715, 1497, 1454, 1390, 1364, 1248, $1170,1100,736,698 \mathrm{~cm}^{-1}$.

${ }^{1} \mathrm{H}$ NMR $\left(500 \mathrm{MHz}, \mathrm{CDCl}_{3}\right): \delta=7.35-7.26(\mathrm{~m}, 15 \mathrm{H}), 4.48(\mathrm{~s}, 6 \mathrm{H}), 4.42$ (br s, $1 \mathrm{H}), 3.45(\mathrm{t}, J=6.4 \mathrm{~Hz}, 6 \mathrm{H}), 1.67-1.65(\mathrm{~m}, 6 \mathrm{H}), 1.60-1.53(\mathrm{~m}$, $6 \mathrm{H}), 1.39(\mathrm{~s}, 9 \mathrm{H})$. 
${ }^{13} \mathrm{C}$ NMR $\left(125 \mathrm{MHz}, \mathrm{CDCl}_{3}\right): \delta=154.1,138.5,128.3,127.6,127.5,78.5$, 72.9, 70.6, 56.9, 31.9, 28.4, 23.7.

HRMS (ESI): $m / z[\mathrm{M}+\mathrm{Na}]^{+}$calcd for $\mathrm{C}_{36} \mathrm{H}_{49} \mathrm{NO}_{5} \mathrm{Na}$ : 598.3508 ; found: 598.350 .

Anal. Calcd for $\mathrm{C}_{36} \mathrm{H}_{49} \mathrm{NO}_{5}$ : C, 75.10; $\mathrm{H}, 8.58 ; \mathrm{N}, 2.43$. Found: C, 74.80; $\mathrm{H}, 8.54 ; \mathrm{N}, 2.60$.

\section{1,7-Bis(benzyloxy)-4-[3-(benzyloxy)propyl]heptan-4-amine (5f)} Synthesized from $\mathbf{9}$ according to the procedure used to prepare $\mathbf{5 c}$. Yield: 266 mg (99\%); yellow oil.

IR (neat): 3061, 3031, 2945, 2855, 1719, 1454, 1361, 1276, $1102 \mathrm{~cm}^{-1}$. ${ }^{1} \mathrm{H}$ NMR $\left(500 \mathrm{MHz}, \mathrm{CDCl}_{3}\right): \delta=7.38-7.26(\mathrm{~m}, 15 \mathrm{H}), 4.49(\mathrm{~s}, 6 \mathrm{H}), 3.45$ $(\mathrm{t}, J=6.6 \mathrm{~Hz}, 6 \mathrm{H}), 1.63-1.57(\mathrm{~m}, 8 \mathrm{H}), 1.41-1.38(\mathrm{~m}, 6 \mathrm{H})$.

${ }^{13} \mathrm{C}$ NMR $\left(125 \mathrm{MHz}, \mathrm{CDCl}_{3}\right): \delta=138.5,128.3,127.6,127.5,72.9,70.9$, 52.8, 36.4, 24.0.

HRMS (ESI): $m / z[\mathrm{M}+\mathrm{Na}]^{+}$calcd for $\mathrm{C}_{31} \mathrm{H}_{41} \mathrm{NO}_{3} \mathrm{Na}$ : 498.2984; found: 498.2968 .

\section{$N, N$-Dibenzyl-1-methyl-2,6,7-trioxabicyclo[2.2.2]octan-4-amine (10)}

To a solution of $3(1.00 \mathrm{~g}, 3.32 \mathrm{mmol})$ in toluene $(6 \mathrm{~mL})$ was added triethyl orthoacetate $(605 \mu \mathrm{L}, 3.32 \mathrm{mmol})$ at $0{ }^{\circ} \mathrm{C}$. After stirring for 45 $\min$ at $80^{\circ} \mathrm{C}$, for $1.25 \mathrm{~h}$ at $100^{\circ} \mathrm{C}$, and for $36 \mathrm{~h}$ at $120^{\circ} \mathrm{C}$, the reaction mixture was evaporated in vacuo. The residue was purified by column chromatography [silica gel PSQ 60B; $n$-hexane- $\mathrm{CHCl}_{3}, 1: 9$ ] to afford 10.

Yield: $536 \mathrm{mg}$ (50\%); white solid; $\mathrm{mp} 122-123{ }^{\circ} \mathrm{C}$.

IR (KBr): 3299, 3063, 2969, 2889, 1952, 1875, 1542, 1255, $1042 \mathrm{~cm}^{-1}$. ${ }^{1} \mathrm{H} \mathrm{NMR}\left(500 \mathrm{MHz}, \mathrm{CDCl}_{3}\right): \delta=7.25-7.19(\mathrm{~m}, 10 \mathrm{H}), 4.07(\mathrm{~s}, 6 \mathrm{H}), 3.76$ (s, $4 \mathrm{H}), 1.42(\mathrm{~s}, 3 \mathrm{H})$.

${ }^{13} \mathrm{C} \mathrm{NMR}\left(125 \mathrm{MHz}, \mathrm{CDCl}_{3}\right): \delta=139.8,128.3,128.1,127.2,108.3,68.6$, $53.8,53.1,22.9$.

\section{1-Methyl-2,6,7-trioxabicyclo[2.2.2]octan-4-amine (5g) ${ }^{11}$}

Synthesized from $\mathbf{1 0}$ according to the procedure used to prepare $\mathbf{5 a}$.

Yield: $16.0 \mathrm{mg}$ (73\%); white solid; $\mathrm{mp} 116-117^{\circ} \mathrm{C}$.

IR (KBr): 3548, 3470, 3414, 3239, 2953, 2893, 2026, 1854, 1734, 1618 $\mathrm{cm}^{-1}$.

${ }^{1} \mathrm{H} \mathrm{NMR}\left(500 \mathrm{MHz}, \mathrm{CDCl}_{3}\right): \delta=3.90(\mathrm{~s}, 6 \mathrm{H}), 1.46$ (s, $\left.3 \mathrm{H}\right), 1.00$ (br s, $2 \mathrm{H})$.

${ }^{13} \mathrm{C}$ NMR $\left(125 \mathrm{MHz}, \mathrm{CDCl}_{3}\right): \delta=108.2,72.7,44.9,22.9$.

Anal. Calcd for $\mathrm{C}_{6} \mathrm{H}_{11} \mathrm{NO}_{3}$ : C, 49.65; H, 7.64; N, 9.65. Found: C, 49.35; H, 7.59; N, 9.46.

\section{Preparation of Isoindoles 11a-f and 11i-k; Typical Procedure}

To a solution of OPA (42.0 mg, $0.313 \mathrm{mmol})$ in anhydrous $\mathrm{MeOH}(5$ $\mathrm{mL}), 1$-propanethiol $(31.0 \mu \mathrm{L}, 0.346 \mathrm{mmol})$ and amine $\mathbf{5 a}(56.5 \mathrm{mg}$, $0.346 \mathrm{mmol}$ ) were added. After stirring in the dark for $3 \mathrm{~h}$ at r.t., the reaction mixture was evaporated in vacuo. The oily residue was purified by column chromatography [silica gel $60 \mathrm{~N}$; $n$-hexane-EtOAc, 9:1 to $3: 1$ ] to afford isoindole 11a $(69.0 \mathrm{mg}, 65 \%)$ accompanied by the formation of isoindolin-1-one 12a (16.0 mg, 18\%).
2-[1,3-Dimethoxy-2-(methoxymethyl)propan-2-yl]-1-(propylthio)-2H-isoindole (11a)

Yield: $69.0 \mathrm{mg}$ (65\%); pale-blue solid; $\mathrm{mp} 67-68{ }^{\circ} \mathrm{C}$.

${ }^{1} \mathrm{H}$ NMR $\left(500 \mathrm{MHz}, \mathrm{CDCl}_{3}\right.$ ): $\delta=7.66$ (br dq, $1 \mathrm{H}$ ), 7.50 (br dt, $1 \mathrm{H}$ ), 7.44 $(\mathrm{d}, J=0.5 \mathrm{~Hz}, 1 \mathrm{H}), 6.99$ (ddd $J=8.5,6.5,0.9 \mathrm{~Hz}, 1 \mathrm{H}), 6.91$ (ddd, $J=$ 8.4, 6.4, $0.9 \mathrm{~Hz}, 1 \mathrm{H}), 4.21$ (s, $6 \mathrm{H}), 3.33(\mathrm{~s}, 9 \mathrm{H}), 2.66$ (t, J = 7.4 Hz, $2 \mathrm{H}$ ), 1.66 (sext, $J=7.5 \mathrm{~Hz}, 2 \mathrm{H}), 1.01(\mathrm{t}, J=7.3 \mathrm{~Hz}, 3 \mathrm{H}$ ).

${ }^{13} \mathrm{C}$ NMR $\left(125 \mathrm{MHz}, \mathrm{CDCl}_{3}\right): \delta=131.7,123.1,122.2,120.9,120.6$, 119.1, 115.7, 109.1, 72.6, 67.8, 59.3, 41.4, 23.0, 13.6.

HRMS (ESI): $m / z$ [M $+\mathrm{Na}]^{+}$calcd for $\mathrm{C}_{18} \mathrm{H}_{27} \mathrm{NO}_{3} \mathrm{SNa}$ : 360.1609 ; found: 360.1608 .

Anal. Calcd for $\mathrm{C}_{18} \mathrm{H}_{27} \mathrm{NO}_{3} \mathrm{~S}$ : C, 64.06; $\mathrm{H}, 8.06 ; \mathrm{N}, 4.15$. Found: $\mathrm{C}, 63.76$; $\mathrm{H}, 8.09 ; \mathrm{N}, 4.10$.

2-[1,3-Diethoxy-2-(ethoxymethyl)propan-2-yl]-1-(propylthio)2H-isoindole (11b)

Yield: $122 \mathrm{mg}$ (85\%); pale-blue solid; $\mathrm{mp} 48-49^{\circ} \mathrm{C}$.

${ }^{1} \mathrm{H}$ NMR (500 MHz, $\mathrm{CDCl}_{3}$ ): $\delta=7.67$ (br dq, $1 \mathrm{H}$ ), 7.53 (d, $J=0.6 \mathrm{~Hz}$, $1 \mathrm{H}$ ), 7.51 (br dt, $1 \mathrm{H}$ ), 6.99 (ddd, $J=8.5,6.4,0.9 \mathrm{~Hz}, 1 \mathrm{H}$ ), 6.91 (ddd, $J=$ 8.4, 6.4, 0.9 Hz, $1 \mathrm{H}), 4.25(\mathrm{~s}, 6 \mathrm{H}), 3.47(\mathrm{q}, J=7.0 \mathrm{~Hz}, 6 \mathrm{H}), 2.66(\mathrm{t}, J=$ $7.4 \mathrm{~Hz}, 2 \mathrm{H}), 1.66$ (sext, $J=7.5 \mathrm{~Hz}, 2 \mathrm{H}), 1.13$ (t, $J=7.0 \mathrm{~Hz}, 9 \mathrm{H}), 1.00$ (t, $J=7.2 \mathrm{~Hz}, 3 \mathrm{H})$.

${ }^{13} \mathrm{C}$ NMR $\left(125 \mathrm{MHz}, \mathrm{CDCl}_{3}\right): \delta=131.7,123.0,122.0,120.6,120.5$, $119.2,116.2,108.9,70.5,68.2,66.9,41.4,23.0,15.1,13.6$.

HRMS (ESI): $m / z$ [M + Na] $]^{+}$calcd for $\mathrm{C}_{21} \mathrm{H}_{33} \mathrm{NO}_{3} \mathrm{SNa}$ : 402.2079; found: 402.2076 .

2-\{6-[(Methoxymethoxy)methyl]-2,4,8,10-tetraoxaundecan-6-yl $\}-$ 1-(propylthio)-2H-isoindole (11c)

Yield: $91.0 \mathrm{mg}$ (57\%); pale-yellow oil.

${ }^{1} \mathrm{H}$ NMR $\left(500 \mathrm{MHz}, \mathrm{CDCl}_{3}\right): \delta=7.67$ (br dq, $\left.1 \mathrm{H}\right), 7.51-7.50(\mathrm{br} \mathrm{m}$, $0.5 \mathrm{H}$ ), 7.49-7.48 (br m, $1.5 \mathrm{H}$ ), 7.00 (ddd, $J=8.5,6.5,0.9 \mathrm{~Hz}, 1 \mathrm{H}$ ), 6.92 (ddd, $J=8.4,6.5,0.9 \mathrm{~Hz}, 1 \mathrm{H}), 4.59(\mathrm{~s}, 6 \mathrm{H}), 4.43(\mathrm{~s}, 6 \mathrm{H}), 3.29(\mathrm{~s}, 9 \mathrm{H})$, 2.71 (t, $J=7.4 \mathrm{~Hz}, 2 \mathrm{H}$ ), 1.67 (sext, $J=7.4 \mathrm{~Hz}, 2 \mathrm{H}$ ), 1.02 (t, $J=7.4 \mathrm{~Hz}$, $3 \mathrm{H})$.

${ }^{13} \mathrm{C}$ NMR $\left(125 \mathrm{MHz}, \mathrm{CDCl}_{3}\right): \delta=131.7,123.1,122.2,121.0,120.4$, 119.2, 115.5, 109.4, 96.8, 67.5, 67.3, 55.5, 41.5, 22.9, 13.6.

HRMS (ESI): $m / z$ [M + Na] ${ }^{+}$calcd for $\mathrm{C}_{21} \mathrm{H}_{33} \mathrm{NO}_{6} \mathrm{SNa}$ : 450.1926; found: 450.1885 .

2-(9-\{[(2-Methoxyethoxy)methoxy]methyl\}-2,5,7,11,13,16-hexaoxaheptadecan-9-yl)-1-(propylthio)-2H-isoindole (11d)

Yield: $113 \mathrm{mg}$ (54\%); pale-yellow oil.

${ }^{1} \mathrm{H}$ NMR $\left(500 \mathrm{MHz}, \mathrm{CDCl}_{3}\right): \delta=7.65(\mathrm{~d}, J=8.6 \mathrm{~Hz}, 1 \mathrm{H}), 7.48(\mathrm{~d}, J=$ $8.3 \mathrm{~Hz}, 1 \mathrm{H}), 7.48$ (s, $1 \mathrm{H}), 7.01-6.98$ (m, $1 \mathrm{H}), 6.93-6.90$ (m, $1 \mathrm{H}), 4.68$ $(\mathrm{s}, 6 \mathrm{H}), 4.43(\mathrm{~s}, 6 \mathrm{H}), 3.56-3.54(\mathrm{~m}, 6 \mathrm{H}), 3.49-3.47(\mathrm{~m}, 6 \mathrm{H}), 3.37(\mathrm{~s}$, $9 \mathrm{H}), 2.69$ (t, $J=7.4 \mathrm{~Hz}, 2 \mathrm{H}), 1.66$ (sext, $J=7.4 \mathrm{~Hz}, 2 \mathrm{H}), 1.01$ (t, $J=$ $7.3 \mathrm{~Hz}, 3 \mathrm{H})$.

${ }^{13} \mathrm{C}$ NMR $\left(125 \mathrm{MHz}, \mathrm{CDCl}_{3}\right): \delta=131.7,123.0,122.3,121.0,120.4$, 119.2, 115.6, 109.4, 95.8, 71.7, 67.7, 67.3, 67.1, 59.0, 41.5, 23.0, 13.6 .

HRMS (ESI): $m / z[\mathrm{M}+\mathrm{Na}]^{+}$calcd for $\mathrm{C}_{27} \mathrm{H}_{45} \mathrm{NO}_{9} \mathrm{SNa}$ : 582.2713 ; found: 582.2701 .

2-(6-\{[(Benzyloxy)methoxy]methyl\}-1,11-diphenyl-2,4,8,10-tetraoxaundecan-6-yl)-1-(propylthio)-2H-isoindole (11e)

Yield: $148 \mathrm{mg}(60 \%)$; orange oil. 
${ }^{1} \mathrm{H}$ NMR $\left(500 \mathrm{MHz}, \mathrm{CDCl}_{3}\right): \delta=7.66(\mathrm{~d}, J=8.6 \mathrm{~Hz}, 1 \mathrm{H}), 7.50(\mathrm{~s}, 1 \mathrm{H})$, 7.47 (d, $J=8.4 \mathrm{~Hz}, 1 \mathrm{H}$ ), 7.33-7.27 ( $\mathrm{m}, 15 \mathrm{H}$ ), 7.01 (ddd, $J=8.5,6.5$, $0.8 \mathrm{~Hz}, 1 \mathrm{H}), 6.93(\mathrm{ddd}, J=8.3,6.5,0.7 \mathrm{~Hz}, 1 \mathrm{H}), 4.72(\mathrm{~s}, 6 \mathrm{H}), 4.52(\mathrm{~s}$, $6 \mathrm{H}$ ), 4.48 (s, $6 \mathrm{H}), 2.66$ (t, J = 7.5 Hz, $2 \mathrm{H}$ ), 1.61 (sext, $J=7.4 \mathrm{~Hz}, 2 \mathrm{H}$ ), $0.94(\mathrm{t}, J=7.4 \mathrm{~Hz}, 3 \mathrm{H})$.

${ }^{13} \mathrm{C}$ NMR $\left(125 \mathrm{MHz}, \mathrm{CDCl}_{3}\right): \delta=137.6,131.8,128.4,127.8,127.7$, 123.1, 122.3, 121.1, 120.4, 119.3, 115.5, 109.6, 94.9, 69.6, 67.7, 67.4, 41.5, 22.9, 13.5 .

HRMS (ESI): $m / z[\mathrm{M}+\mathrm{Na}]^{+}$calcd for $\mathrm{C}_{39} \mathrm{H}_{45} \mathrm{NO}_{6} \mathrm{SNa}$ : 678.2865 ; found: 678.2883.

\section{2-\{1,7-Bis(benzyloxy)-4-[3-(benzyloxy)propyl]heptan-4-yl\}-1-} (propylthio)-2H-isoindole (11f)

Yield: $224 \mathrm{mg}$ (93\%); pale-yellow oil.

${ }^{1} \mathrm{H}$ NMR $\left(500 \mathrm{MHz}, \mathrm{CDCl}_{3}\right): \delta=7.68(\mathrm{~d}, J=8.5 \mathrm{~Hz}, 1 \mathrm{H}), 7.48(\mathrm{~d}, J=$ $8.4 \mathrm{~Hz}, 1 \mathrm{H}), 7.39(\mathrm{~s}, 1 \mathrm{H}), 7.33-7.25(\mathrm{~m}, 15 \mathrm{H}), 7.03-7.00(\mathrm{~m}, 1 \mathrm{H})$, 6.94-6.91 (m, $1 \mathrm{H}), 4.44(\mathrm{~s}, 6 \mathrm{H}), 3.42(\mathrm{t}, J=6.3 \mathrm{~Hz}, 6 \mathrm{H}), 2.65(\mathrm{t}, J=$ $7.4 \mathrm{~Hz}, 2 \mathrm{H}$ ), 2.43-2.40 (m, $6 \mathrm{H}), 1.60$ (sext, $J=7.4 \mathrm{~Hz}, 2 \mathrm{H}), 1.40-1.34$ $(\mathrm{m}, 6 \mathrm{H}), 0.94(\mathrm{t}, J=7.4 \mathrm{~Hz}, 3 \mathrm{H})$.

${ }^{13} \mathrm{C}$ NMR $\left(125 \mathrm{MHz}, \mathrm{CDCl}_{3}\right): \delta=138.4,131.9,128.3,127.6,127.5$, 122.5, 121.9, 120.8, 120.3, 119.2, 114.3, 109.7, 72.9, 70.2, 66.9, 41.5, 32.9, 23.7, 22.8, 13.6 .

HRMS (ESI): $m / z[\mathrm{M}+\mathrm{Na}]^{+}$calcd for $\mathrm{C}_{42} \mathrm{H}_{51} \mathrm{NO}_{3} \mathrm{SNa}$ : 672.3487 ; found: 672.3490 .

Anal. Calcd for $\mathrm{C}_{42} \mathrm{H}_{51} \mathrm{NO}_{3} \mathrm{~S}$ : C, 77.62; H, 7.91; N, 2.16. Found: C, 77.32; $\mathrm{H}, 7.98 ; \mathrm{N}, 2.13$.

\section{4-(3-Hydroxypropyl)-4-[1-(propylthio)-2H-isoindol-2-yl]heptane- 1,7-diol (11i)}

Yield: $127 \mathrm{mg}$ (90\%); mp $116-118{ }^{\circ} \mathrm{C}$ (pale-yellow needles, $\mathrm{CHCl}_{3} / n$ hexane).

${ }^{1} \mathrm{H} \mathrm{NMR}\left(500 \mathrm{MHz}, \mathrm{CDCl}_{3}\right): \delta=7.69(\mathrm{dd}, J=8.5,0.8 \mathrm{~Hz}, 1 \mathrm{H}), 7.50(\mathrm{dt}$, $J=8.4,0.9 \mathrm{~Hz}, 1 \mathrm{H}$ ), $7.43(\mathrm{~s}, 1 \mathrm{H}), 7.03$ (ddd, $J=8.5,6.5,0.9 \mathrm{~Hz}, 1 \mathrm{H}$ ), 6.94 (ddd, $J=8.4,6.5,0.9 \mathrm{~Hz}, 1 \mathrm{H}), 3.62(\mathrm{t}, J=6.2 \mathrm{~Hz}, 6 \mathrm{H}), 2.71(\mathrm{t}, J=$ $7.4 \mathrm{~Hz}, 2 \mathrm{H}$ ), 2.45-2.42 (m, $6 \mathrm{H}$ ), 1.69 (sext, $J=7.4 \mathrm{~Hz}, 2 \mathrm{H}$ ), 1.39 (br s, $3 \mathrm{H}), 1.36-1.30(\mathrm{~m}, 6 \mathrm{H}), 1.03(\mathrm{t}, J=7.4 \mathrm{~Hz}, 3 \mathrm{H})$.

${ }^{13} \mathrm{C}$ NMR $\left(125 \mathrm{MHz}, \mathrm{CDCl}_{3}\right): \delta=131.9,122.6,122.2,121.0,120.3$, $119.1,114.3,109.5,66.9,62.5,41.6,32.5,26.4,22.9,13.7$.

HRMS (ESI): $m / z$ [M + Na] $]^{+}$calcd for $\mathrm{C}_{21} \mathrm{H}_{33} \mathrm{NO}_{3} \mathrm{SNa}$ : 402.2079; found: 402.2067.

2-\{1,7-Bis(benzyloxy)-4-[3-(benzyloxy)propyl]heptan-4-yl\}-1-(octylthio)-2H-isoindole (11j)

Yield: $206 \mathrm{mg}$ (96\%); pale-yellow oil.

$\left.{ }^{1} \mathrm{H} \mathrm{NMR} \mathrm{(500} \mathrm{MHz,} \mathrm{CDCl}\right)$ ): $\delta=7.67$ (br d, $1 \mathrm{H}$ ), 7.48 (br d, $\left.1 \mathrm{H}\right), 7.38(\mathrm{~s}$, $1 \mathrm{H}), 7.33-7.24(\mathrm{~m}, 15 \mathrm{H}), 7.01$ (ddd, $J=8.5,6.5,0.9 \mathrm{~Hz}, 1 \mathrm{H}), 6.93$ (ddd, $J=8.4,6.5,0.8 \mathrm{~Hz}, 1 \mathrm{H}), 4.44(\mathrm{~s}, 6 \mathrm{H}), 3.42(\mathrm{t}, J=6.3 \mathrm{~Hz}, 6 \mathrm{H}), 2.67$ (t, $J=7.4 \mathrm{~Hz}, 2 \mathrm{H}), 2.43-2.40$ (m, $6 \mathrm{H}), 1.61-1.54$ (m, $2 \mathrm{H}), 1.39-1.24$ $(\mathrm{m}, 16 \mathrm{H}), 0.88(\mathrm{t}, J=7.0 \mathrm{~Hz}, 3 \mathrm{H})$.

${ }^{13} \mathrm{C}$ NMR $\left(125 \mathrm{MHz}, \mathrm{CDCl}_{3}\right): \delta=138.4,131.8,128.3,127.55,127.48$, 122.6, 121.9, 120.8, 120.3, 119.2, 114.2, 109.8, 72.9, 70.2, 66.9, 39.6, $32.9,31.8,29.4,29.3,29.2,29.0,23.7,22.7,14.1$.

HRMS (ESI): $m / z$ [M $+\mathrm{Na}]^{+}$calcd for $\mathrm{C}_{47} \mathrm{H}_{61} \mathrm{NO}_{3} \mathrm{SNa}$ : 742.4270; found: 742.4276 .
2-\{1,7-Bis(benzyloxy)-4-[3-(benzyloxy)propyl]heptan-4-yl\}-1-(dodecylthio)-2H-isoindole (11k)

Yield: $210 \mathrm{mg}$ (91\%); pale-yellow oil.

${ }^{1} \mathrm{H}$ NMR $\left(500 \mathrm{MHz}, \mathrm{CDCl}_{3}\right): \delta=7.67(\mathrm{~d}, J=8.6 \mathrm{~Hz}, 1 \mathrm{H}), 7.48(\mathrm{~d}, J=$ $8.4 \mathrm{~Hz}, 1 \mathrm{H}), 7.38(\mathrm{~s}, 1 \mathrm{H}), 7.33-7.24(\mathrm{~m}, 15 \mathrm{H}), 7.03-7.00(\mathrm{~m}, 1 \mathrm{H})$, 6.94-6.91 (m, $1 \mathrm{H}), 4.44(\mathrm{~s}, 6 \mathrm{H}), 3.42(\mathrm{t}, J=6.3 \mathrm{~Hz}, 6 \mathrm{H}), 2.67(\mathrm{t}, J=$ $7.4 \mathrm{~Hz}, 2 \mathrm{H}), 2.43-2.40(\mathrm{~m}, 6 \mathrm{H}), 1.60-1.54(\mathrm{~m}, 2 \mathrm{H}), 1.38-1.25(\mathrm{~m}$, $24 \mathrm{H}), 0.88(\mathrm{t}, J=6.9 \mathrm{~Hz}, 3 \mathrm{H})$.

${ }^{13} \mathrm{C}$ NMR $\left(125 \mathrm{MHz}, \mathrm{CDCl}_{3}\right): \delta=138.4,131.8,128.3,127.55,127.47$, 122.6, 121.9, 120.8, 120.3, 119.2, 114.2, 109.8, 72.9, 70.2, 66.9, 39.6, $32.9,31.9,29.7,29.64,29.56,29.5,29.38,29.36,29.1,29.0,23.7,22.7$, 14.1.

HRMS (ESI): $m / z[\mathrm{M}+\mathrm{Na}]^{+}$calcd for $\mathrm{C}_{51} \mathrm{H}_{69} \mathrm{NO}_{3} \mathrm{SNa}$ : 798.4896; found: 798.4892 .

Anal. Calcd for $\mathrm{C}_{51} \mathrm{H}_{69} \mathrm{NO}_{3} \mathrm{~S}$ : C, 78.92; $\mathrm{H}, 8.96 ; \mathrm{N}, 1.80$. Found: C, 78.62; $\mathrm{H}, 9.01 ; \mathrm{N}, 1.83$.

2-[1,3-Dimethoxy-2-(methoxymethyl)propan-2-yl]isoindolin-1one (12a)

Yield: $16.0 \mathrm{mg}$ (18\%); white solid; $\mathrm{mp} 63.5-66.0{ }^{\circ} \mathrm{C}$.

IR (KBr): 2976, 2876, 1684, 1471, 1383, 1304, $1103 \mathrm{~cm}^{-1}$.

${ }^{1} \mathrm{H}$ NMR $\left(500 \mathrm{MHz}, \mathrm{CDCl}_{3}\right): \delta=7.78(\mathrm{~d}, J=7.5 \mathrm{~Hz}, 1 \mathrm{H}), 7.51$ (td, $J=7.4$, $1.1 \mathrm{~Hz}, 1 \mathrm{H}), 7.44-7.39(\mathrm{~m}, 2 \mathrm{H}), 4.66(\mathrm{~s}, 2 \mathrm{H}), 3.97$ (s, $6 \mathrm{H}), 3.35$ (s, $9 \mathrm{H})$.

${ }^{13} \mathrm{C}$ NMR $\left(125 \mathrm{MHz}, \mathrm{CDCl}_{3}\right): \delta=169.6,142.1,133.5,131.1,127.6$, $123.2,122.3,71.1,63.4,59.3,50.1$.

HRMS (ESI): $m / z[\mathrm{M}+\mathrm{Na}]^{+}$calcd for $\mathrm{C}_{15} \mathrm{H}_{21} \mathrm{NO}_{4} \mathrm{Na}$ : 302.1368; found: 302.1342 .

\section{2-[1,3-Diethoxy-2-(ethoxymethyl)propan-2-yl]isoindolin-1-one (12b)}

Yield: $14.0 \mathrm{mg}$ (12\%); yellow oil.

IR (neat): 2974, 2870, 1684, 1469, 1393, 1301, $1103 \mathrm{~cm}^{-1}$.

${ }^{1} \mathrm{H}$ NMR $\left(500 \mathrm{MHz}, \mathrm{CDCl}_{3}\right): \delta=7.78(\mathrm{~d}, J=7.5 \mathrm{~Hz}, 1 \mathrm{H}), 7.50$ (td, $J=7.4$, $1.1 \mathrm{~Hz}, 1 \mathrm{H}), 7.43-7.39(\mathrm{~m}, 2 \mathrm{H}), 4.71(\mathrm{~s}, 2 \mathrm{H}), 4.03(\mathrm{~s}, 6 \mathrm{H}), 3.50(\mathrm{q}, J=$ $7.0 \mathrm{~Hz}, 6 \mathrm{H}), 1.14(\mathrm{t}, J=7.0 \mathrm{~Hz}, 9 \mathrm{H})$.

${ }^{13} \mathrm{C}$ NMR $\left(125 \mathrm{MHz}, \mathrm{CDCl}_{3}\right): \delta=169.6,142.2,133.7,130.9,127.4$, $123.2,122.2,68.9,66.8,63.6,50.3,15.2$.

HRMS (ESI): $m / z$ [M $+\mathrm{Na}]^{+}$calcd for $\mathrm{C}_{18} \mathrm{H}_{27} \mathrm{NO}_{4} \mathrm{Na}$ : 344.1838; found: 344.1834 .

2-\{6-[(Methoxymethoxy)methyl]-2,4,8,10-tetraoxaundecan-6yl\}isoindolin-1-one (12c)

Yield: $57.2 \mathrm{mg}$ (41\%); pale-yellow oil.

IR (neat): 2947, 2890, 2823, 1684, 1470, 1152, 1111, $1041 \mathrm{~cm}^{-1}$.

${ }^{1} \mathrm{H}$ NMR $\left(500 \mathrm{MHz}, \mathrm{CDCl}_{3}\right): \delta=7.80(\mathrm{~d}, J=7.6 \mathrm{~Hz}, 1 \mathrm{H}), 7.52(\mathrm{td}, J=7.5$, $1.1 \mathrm{~Hz}, 1 \mathrm{H}), 7.45-7.39(\mathrm{~m}, 2 \mathrm{H}), 4.69(\mathrm{~s}, 2 \mathrm{H}), 4.64(\mathrm{~s}, 6 \mathrm{H}), 4.17(\mathrm{~s}$, $6 \mathrm{H}), 3.33(\mathrm{~s}, 9 \mathrm{H})$.

${ }^{13} \mathrm{C}$ NMR $\left(125 \mathrm{MHz}, \mathrm{CDCl}_{3}\right): \delta=169.5,141.9,133.4,131.2,127.7$, $123.3,122.3,96.9,66.2,62.8,55.4,50.0$.

HRMS (ESI): $m / z$ [M $+\mathrm{Na}]^{+}$calcd for $\mathrm{C}_{18} \mathrm{H}_{27} \mathrm{NO}_{7} \mathrm{Na}$ : 392.1685; found: 392.1664 .

2-(9-\{[(2-Methoxyethoxy)methoxy]methyl\}-2,5,7,11,13,16-hexaoxaheptadecan-9-yl)isoindolin-1-one (12d)

Yield: $83.2 \mathrm{mg}$ (44\%); pale-yellow oil. 
IR (neat): 2930, 2886, 2819, 1685, 1470, 1452, 1118, $1046 \mathrm{~cm}^{-1}$. ${ }^{1} \mathrm{H} \mathrm{NMR}\left(500 \mathrm{MHz}, \mathrm{CDCl}_{3}\right): \delta=7.78(\mathrm{~d}, J=7.6 \mathrm{~Hz}, 1 \mathrm{H}), 7.52(\mathrm{td}, J=7.5$, $1.1 \mathrm{~Hz}, 1 \mathrm{H}), 7.45-7.38(\mathrm{~m}, 2 \mathrm{H}), 4.72(\mathrm{~s}, 6 \mathrm{H}), 4.66(\mathrm{~s}, 2 \mathrm{H}), 4.17$ (s, $6 \mathrm{H}), 3.64-3.62(\mathrm{~m}, 6 \mathrm{H}), 3.53-3.50(\mathrm{~m}, 6 \mathrm{H}), 3.37(\mathrm{~s}, 9 \mathrm{H})$.

${ }^{13} \mathrm{C}$ NMR $\left(125 \mathrm{MHz}, \mathrm{CDCl}_{3}\right): \delta=169.4,141.9,133.3,131.2,127.7$, $123.2,122.3,96.0,71.7,67.0,66.3,62.8,59.0,50.0$.

HRMS (ESI): $m / z[\mathrm{M}+\mathrm{Na}]^{+}$calcd for $\mathrm{C}_{24} \mathrm{H}_{39} \mathrm{NO}_{10} \mathrm{Na}$ : 524.2472 ; found: 524.2473.

2-(6-\{[(Benzyloxy)methoxy]methyl\}-1,11-diphenyl-2,4,8,10-tetraoxaundecan-6-yl)isoindolin-1-one (12e)

Yield: $87.0 \mathrm{mg}$ (39\%); pale-yellow oil.

IR (neat): 2943, 2886, 1684, 1454, 1383, 1169, 1112, $1044 \mathrm{~cm}^{-1}$.

${ }^{1} \mathrm{H} \mathrm{NMR}\left(500 \mathrm{MHz}, \mathrm{CDCl}_{3}\right): \delta=7.79(\mathrm{~d}, J=7.6 \mathrm{~Hz}, 1 \mathrm{H}), 7.51(\mathrm{td}, J=7.5$, $1.1 \mathrm{~Hz}, 1 \mathrm{H}), 7.42(\mathrm{t}, J=7.5 \mathrm{~Hz}, 1 \mathrm{H}), 7.37(\mathrm{~d}, J=7.5 \mathrm{~Hz}, 1 \mathrm{H}), 7.34-7.26$ $(\mathrm{m}, 15 \mathrm{H}), 4.76(\mathrm{~s}, 6 \mathrm{H}), 4.66(\mathrm{~s}, 2 \mathrm{H}), 4.55(\mathrm{~s}, 6 \mathrm{H}), 4.26(\mathrm{~s}, 6 \mathrm{H})$.

${ }^{13} \mathrm{C}$ NMR $\left(125 \mathrm{MHz}, \mathrm{CDCl}_{3}\right): \delta=169.5,141.8,137.7,133.3,131.2$, 128.4, 127.8, 127.73, 127.67, 123.3, 122.3, 95.1, 69.5, 66.5, 62.9, 50.0.

HRMS (ESI): $m / z[\mathrm{M}+\mathrm{Na}]^{+}$calcd for $\mathrm{C}_{36} \mathrm{H}_{39} \mathrm{NO}_{7} \mathrm{Na}$ : 620.2624; found: 620.2635 .

\section{2-\{1,7-Bis(benzyloxy)-4-[3-(benzyloxy)propyl]heptan-4-yl\}isoin-} dolin-1-one (12f)

Yield: $13.0 \mathrm{mg}$ (ca. 6\%); pale-yellow oil.

${ }^{1} \mathrm{H} \mathrm{NMR}\left(500 \mathrm{MHz}, \mathrm{CDCl}_{3}\right): \delta=7.77(\mathrm{~d}, J=7.5 \mathrm{~Hz}, 1 \mathrm{H}), 7.49$ (td, $J=7.4$, $1.2 \mathrm{~Hz}, 1 \mathrm{H}), 7.43(\mathrm{~d}, J=7.4 \mathrm{~Hz}, 1 \mathrm{H}), 7.36-7.22(\mathrm{~m}, 16 \mathrm{H}), 4.47(\mathrm{~s}, 2 \mathrm{H})$, $4.46(\mathrm{~s}, 6 \mathrm{H}), 3.46(\mathrm{t}, J=6.7 \mathrm{~Hz}, 6 \mathrm{H}), 2.13-2.05(\mathrm{~m}, 6 \mathrm{H}), 1.62-1.52(\mathrm{~m}$, $6 \mathrm{H})$.

HRMS (ESI): $m / z[\mathrm{M}+\mathrm{Na}]^{+}$calcd for $\mathrm{C}_{39} \mathrm{H}_{45} \mathrm{NO}_{4} \mathrm{Na}$ : 614.3246 ; found: 614.3204.

\section{HPLC Equipment and Conditions}

A Gulliver HPLC system (JASCO) equipped with a Rheodyne 7125 injection valve (20 $\mu \mathrm{L}$ loop), UV/Vis detector (UV-970), and HPLC pump (PU-980) was used. HPLC analysis was performed with a TSKgel ODS80Ts analytical column $(250 \times 4.6 \mathrm{~mm}$ id, $5 \mu \mathrm{m}$, Tosoh $)$ by measuring absorbance at $330 \mathrm{~nm}$. The mobile phase was a mixture of $\mathrm{MeOH}$ and water $(200: 1, \mathrm{v} / \mathrm{v})$ at a flow rate of $1.0 \mathrm{~mL} \mathrm{~min}^{-1}$.

\section{Supporting Information}

Supporting information for this article is available online at https://doi.org/10.1055/s-0036-1591932.

\section{References}

(1) Burford, K. N. Isoindole, In e-EROS Encyclopedia of Reagents for Organic Synthesis; Wiley: New York, 2015.

(2) (a) Roth, M. Anal. Chem. 1971, 43, 880. (b) Zuman, P. Chem. Rev. 2004, 104, 3217.
(3) (a) Liu, H. In Methods in Molecular Biology; Cooper, C.; Packer, N.; Williams, K., Eds.; Humana Press: Totowa, NJ, 2001, 123. (b) Hermanson, G. T. In Bioconjugate Techniques, 2nd ed.; Elsevier: London, 2008, 128.

(4) (a) Gardner, W. S.; Miller, W. H. Anal. Biochem. 1980, 101, 61. (b) Godel, H.; Graser, T.; Földi, P.; Pfaender, P.; Fürst, P. J. Chromatogr. A 1984, 297, 49. (c) Lookhart, G. L.; Jones, B. L. Cereal Chem. 1985, 62, 97. (d) Dorresteijn, R. C.; Berwald, L. G.; Zomer, G.; de Gooijer, C. D.; Wieten, G.; Beuvery, E. C. J. Chromatogr. A 1996, 724, 159. (e) Lázaro de la Torre, C. A.; Conte-Júnior, C. A. Braz. J. Vet. Res. Anim. Sci. 2013, 50, 430. (f) Borowczyk, K.; Chwatko, G.; Kubalczyk, P.; Jakubowski, H.; Kubalska, J.; Głowacki, R. Talanta 2016, 161, 917. (g) Douša, M.; Pivoňková, V.; Sýkora, D. J. Sep. Sci. 2016, 39, 3145.

(5) (a) Nakamura, H.; Matsumoto, A.; Tamura, Z. Anal. Lett. 1982, 15, 1393. (b) Stobaugh, J. F.; Repta, A. J.; Sternson, L. A.; Garren, K. W. Anal. Biochem. 1983, 135, 495. (c) Jacobs, W. A.; Leburg, M. W.; Madaj, E. J. Anal. Biochem. 1986, 156, 334. (d) Stobaugh, J. F.; Repta, A. J.; Sternson, L. A. J. Pharm. Biomed. Anal. 1986, 4, 341. (e) García Alvarez-Coque, M. C.; Medina Hernández, M. J.; Villanueva Camañas, R. M.; Mongay Fernández, C. Anal. Biochem. 1989, 178, 1. (f) Dai, F.; Burkert, V. P.; Singh, H. N.; Hinze, W. L. Microchem. J. 1997, 57, 166. (g) Heugebaert, T. S. A.; Roman, B. I.; Stevens, C. V. Chem. Soc. Rev. 2012, 41, 5626.

(6) (a) West, R.; Fink, M. J.; Michl, J. Science 1981, 214, 1343. (b) Yoshifuji, M.; Shima, I.; Inamoto, N.; Hirotsu, K.; Higuchi, T. J. Am. Chem. Soc. 1981, 103, 4587.

(7) (a) Okazaki, R.; West, R. Adv. Organomet. Chem. 1996, 39, 231. (b) Power, P. P. Chem. Rev. 1999, 99, 3463. (c) Tokitoh, N. Acc. Chem. Res. 2004, 37, 86. (d) Kira, M.; Iwamoto, T. Adv. Organomet. Chem. 2006, 54, 73. (e) Mizuhata, Y.; Sasamori, T.; Tokitoh, N. Chem. Rev. 2009, 109, 3479. (f) Wang, Y.; Robinson, G. H. Chem. Commun. 2009, 5201. (g) Fischer, R. C.; Power, P. P. Chem. Rev. 2010, 110, 3877. (h) Scheschkewitz, D. Chem. Lett. 2011, 40, 2. (i) Asay, M.; Sekiguchi, A. Bull. Chem. Soc. Jpn. 2012, 85, 1245. (j) Sasamori, T.; Tokitoh, N. Bull. Chem. Soc. Jpn. 2013, 86, 1005. (k) Yoshifuji, M. Eur. J. Inorg. Chem. 2016, 607. (l) Yoshifuji, M. Phosphorus, Sulfur, Silicon Relat. Elem. 2016, 191, 1452.

(8) (a) Sipos, A.; Török, Z.; Röth, E.; Kiss-Szikszai, A.; Batta, G.; Bereczki, I.; Fejes, Z.; Borbás, A.; Ostorházi, E.; Rozgonyi, F.; Naesens, L.; Herczegh, P. Bioorg. Med. Chem. Lett. 2012, 22, 7092. (b) Sipos, A.; Máté, G.; Röth, E.; Borbás, A.; Batta, G.; Bereczki, I.; Kéki, S.; Jóna, I.; Ostorházi, E.; Rozgonyi, F.; Vanderlinden, E.; Naesens, L.; Herczegh, P. Eur. J. Med. Chem. 2012, 58, 361.

(9) (a) Tonhauser, C.; Schüll, C.; Dingels, C.; Frey, H. ACS Macro Lett. 2012, 1, 1094. (b) Schüll, C.; Nuhn, L.; Mangold, C.; Christ, E.; Zentel, R.; Frey, H. Macromolecules 2012, 45, 5901.

(10) (a) Segura, M.; Sansone, F.; Casnati, A.; Ungaro, R. Synthesis 2001, 2105. (b) Pasha, A.; Lin, M.; Tircsó, G.; Rostollan, C. L.; Woods, M.; Kiefer, G. E.; Sherry, A. D.; Sun, X. J. Biol. Inorg. Chem. 2009, 14, 421. (c) Chen, Z.; Hu, W.; Wang, M.; Wang, L.; Su, G.; Wang, J. Carbohydr. Res. 2016, 429, 81. (d) Das, R.; Mukhopadhyay, B. Tetrahedron Lett. 2016, 57, 1775.

(11) Bundy, G. L.; Pals, D. T.; Lawson, J. A.; Couch, S. J.; Lipton, M. F.; Mauragis, M. A. J. Med. Chem. 1990, 33, 2276. 